\title{
Real-Time Discrimination of Phases with Similar Kikuchi patterns but Different Chemistry through Simultaneous EBSD and EDS
}

\author{
J. Goulden ${ }^{1}$, S. Sitzman ${ }^{2}$, K. Larsen ${ }^{1}$, and R. Jones ${ }^{1}$ \\ ${ }^{1}$ Oxford Instruments NanoAnalysis, Halifax Road, High Wycombe, HP12 3SE, UK \\ ${ }^{2}$ Oxford Instruments America, Concord, MA, USA
}

EBSD is a widely accepted, powerful analytical technique capable of a wide variety of microstructural analyses, including preferred crystallographic orientation, grain size, grain boundary characterization, phase distribution and inter-phase orientation relationship determination. The technique is adept at discriminating phases crystallographically, so crystallography-based phase identification and discrimination during mapping are powerful applications of EBSD. However, limitations exist, and there are cases where indexing by conventional inter-band angle matching alone fails to discriminate phases yielding very similar Kikuchi patterns, for example, in the case of samples containing more than one face centered cubic metal phase.

A number of different solutions have been applied to solve this, both in real-time and in post-acquisition. In one real-time method, Kikuchi band width matching is used to exploit inter-planar spacing differences between phases. With high accuracy band edge detection methods, reliable comparisons between measured and calculated bandwidths for the phases involved are used to determine a best match. This method is useful for band width differences of about $10 \%$ or greater.

Another real-time alternative is phase differentiation on the basis of chemistry, in which no minimum difference in crystal structure or unit cell parameter is necessary for discrimination. This technique uses EDS data acquired simultaneously with EBSD data for each point analyzed. When more than one viable solution results from indexing alone, EDS information for the point is used to weigh the results in favor of the solution whose EDS data best match reference information for the phases involved.

This is illustrated in the example below. A hole in a copper gasket has been filled with solder, and the heat applied to melt the solder has caused a reaction between it and the surrounding copper. The associated phase changes were investigated by EBSD. The sample contains copper, lead, tin and copper-tin intermetallic phases. Copper and lead possess the same face-centered cubic crystal structure (space group 225), thus show the same Kikuchi bands and inter-band angles.

Conventional EBSD indexing cannot tell these phases apart. Adding automatic band width matching gives a reasonable improvement in copper-lead discrimination (Fig. 1a), even when only moderate pattern quality settings are used for reasonable acquisition speeds under normal conditions. Simultaneously collected EDS maps (Fig.s 1b, 1c and 1d) indicate that the phases present show very different chemistries. AZtec TruPhase exploits these chemical differences to yield almost perfect differentiation between the copper and lead phases, as seen in Fig. 1e, and does not require band width matching. This poster will discuss this technique and its application to other samples. 


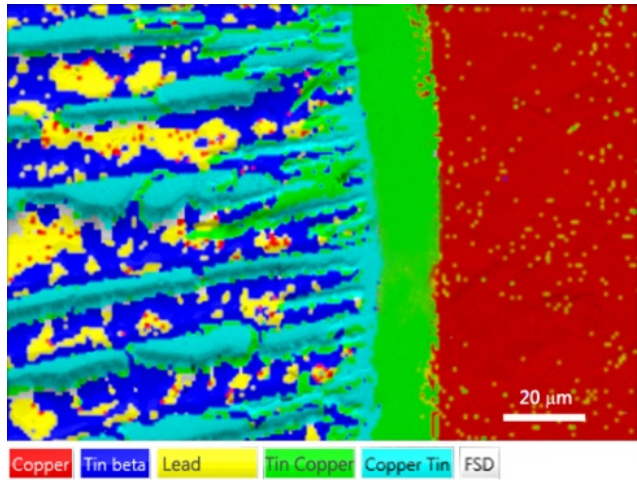

Figure 1a. Phase map from indexing including band width matching, showing reasonable phase discrimination with some remaining errors between the red $\mathrm{Cu}$ and yellow $\mathrm{Pb}$ phases.

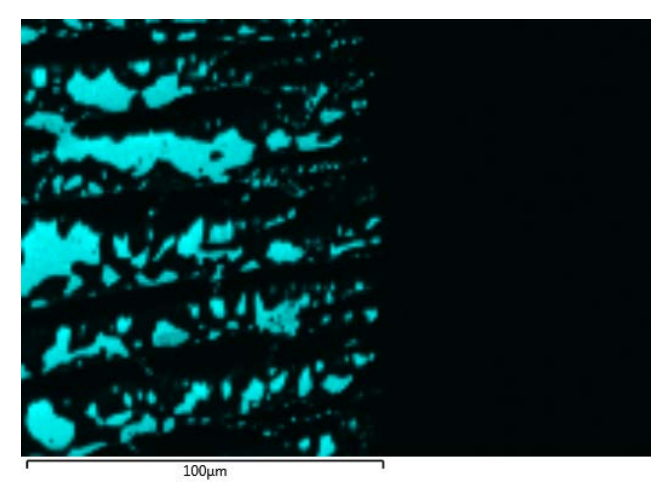

Figure 1c. $\mathrm{Pb} \mathrm{X}$-ray map. Note the lack of $\mathrm{Pb}$ in the copper region on the right.

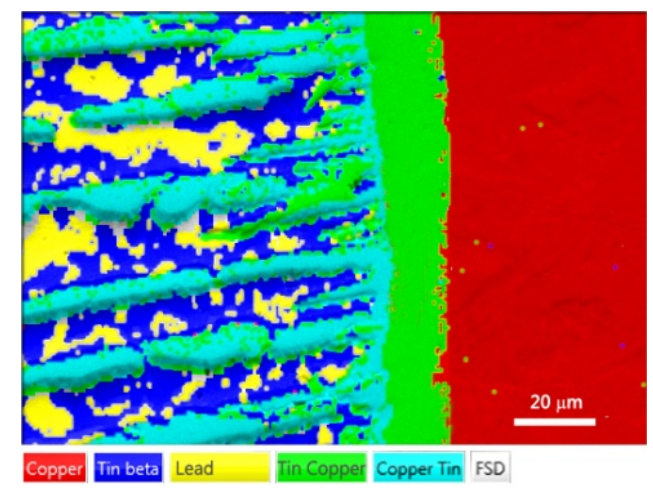

Figure 1e. EBSD phase map, collected with Aztec Truphase activated during indexing, shows almost perfect discrimination of the lead and copper phases.

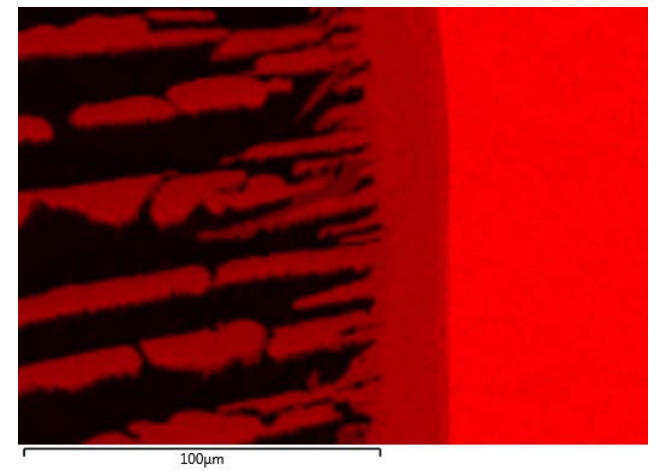

Figure 1b. Cu X-ray map. Note the lack of $\mathrm{Cu}$ in the $\mathrm{Pb}+\mathrm{Sn}$ bands on the left side of the map.

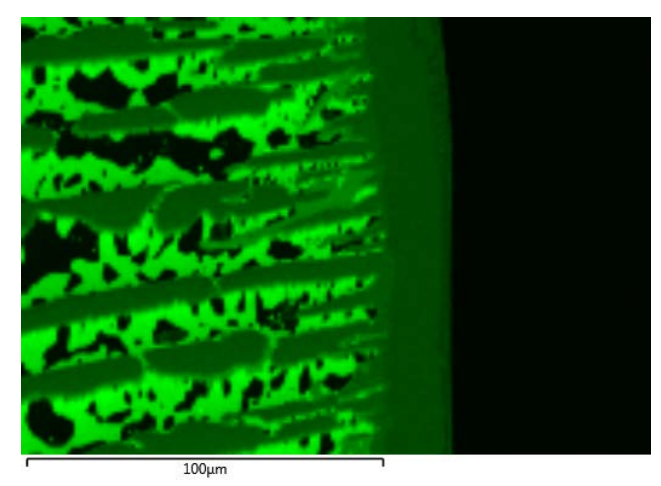

Figure 1d. Sn X-ray map. 